\title{
Teores foliares de nutrientes em doze genótipos de videira
}

\section{Levels of nutrients in the leaves of twelve genotypes of grape}

\author{
Patrícia Gomes de Oliveira Pessanha ${ }^{1 *}$; Alexandre Pio Viana ${ }^{2}$; \\ Almy Junior Cordeiro de Carvalho ${ }^{3}$; Jurandi Gonçalves de Oliveira ${ }^{4}$
}

\section{Resumo}

O objetivo deste trabalho foi o de caracterizar o teor de nutrientes minerais: $\mathrm{N}, \mathrm{P}, \mathrm{K}, \mathrm{Ca}, \mathrm{Mg}, \mathrm{S}, \mathrm{Mn}$, $\mathrm{Fe}, \mathrm{Zn}, \mathrm{Cu}$, Mo e B de doze genótipos de videira: 'Itália', 'Rubi', 'Patrícia', 'Isabel', 'Redglobe', 'Roberta', 'Kyoho', 'Romana', 'Rosalinda', 'Moscatel de Hamburgo', 'Niágara Rosada' e 'UVF 01' na região Norte Fluminense. Realizou-se a poda, seguida de adubação convencional e orgânica e análise do limbo foliar em duas épocas do ciclo reprodutivo da videira: no pleno florescimento e início da maturação das bagas ("veraison"). Nessa caracterização, concluiu-se que o limbo foliar coletado refletiu bem o estado nutricional dos genótipos de videira. Os genótipos não apresentaram níveis de $\mathrm{N}$ abaixo do normal, sendo a maioria dos genótipos apresentaram níveis normais. Nenhum genótipo apresentou níveis abaixo do normal de K e P. Todos os genótipos apresentaram níveis de $\mathrm{Ca}$ abaixo da faixa normal para as duas épocas amostradas e níveis de $\mathrm{Mg}$ na faixa normal. Todos os genótipos apresentaram níveis normais de B.

Palavra-chave: Vitis, nutrição mineral, análise foliar

\begin{abstract}
The objective of this study was to characterize the levels of mineral nutrients: $\mathrm{N}, \mathrm{P}, \mathrm{K}, \mathrm{Ca}, \mathrm{Mg}, \mathrm{S}, \mathrm{Mn}$, $\mathrm{Fe}, \mathrm{Zn}, \mathrm{Cu}$, Mo and B of twelve genotypes of grapevine: 'Italy', 'Ruby', 'Patricia', 'Isabel', 'Redglobe', 'Roberta', 'Kyoho', 'Roman', 'Rosalinda', 'Moscatel de Hamburgo', 'Niagara Rosada' and 'UVF 01' in the North of Rio de Janeiro State. The plants were pruinned and fertilized (organic and mineral). The leaf blades were analyzed in two periods of the reproductive cycle of the vine: in full bloom and the beginning of berry ripening (veraison). In this characterization, it was concluded that the leaves reflected the nutritional status of the vine genotypes. The genotypes did not show levels of $\mathrm{N}$ below normal, with most genotypes showing normal levels. Neither genotype showed below normal levels of K and P. All genotypes showed levels of $\mathrm{Ca}$ below the normal range for all seasons and $\mathrm{Mg}$ in the normal range. All genotypes showed normal levels of B.
\end{abstract}

Key words: Vitis, nutrition, foliar analysis

${ }^{1}$ Eng $^{\mathrm{a}}$ Agr ${ }^{\circ}$, M.Sc. Campos, RJ, Universidade Estadual Norte Fluminense, UENF/CCTA/LMGV, CEP 28015-620, Fone: 22 2731 7900. E-mail: patriciagop@uenf.br

${ }^{2}$ Eng $^{\mathrm{a}}$ Agr $^{\circ}$, D. Sc., Professor da Universidade Estadual Norte Fluminense, UENF/CCTA/LMGV, CEP 28015-620. E-mail: pirapora@uenf.br

${ }^{3}$ Eng $^{\mathrm{a}}$ Agr $^{\circ}$, D. Sc., Professor da Universidade Estadual Norte Fluminense, UENF/CCTA/LFIT, CEP 28015-620. E-mail:almy@, uenf.br

${ }^{4}$ Enga $^{\mathrm{a}}$ Agr ${ }^{\circ}$, D. Sc., Professor da Universidade Estadual Norte Fluminense, UENF/CCTA/LMGV, CEP 28015-620. E-mail: jugo@ uenf.br

* Autor para correspondência 
A região Norte-Noroeste Fluminense, marcada pelo cultivo intensivo da cana-de-açúcar, apresenta uma forte vocação para fruticultura. Sua localização próxima aos grandes centros consumidores da região Sudeste facilita a comercialização e tende a incrementar os ganhos com os produtos vendidos.

A cultura da videira tem sido apontada como uma das mais promissoras para a região, pela sua fácil adaptação edafoclimática e pelos elevados preços que seus frutos alcançam no mercado, com conseqüência interesse por parte dos produtores.

Para a análise foliar em videira há muitas divergências quanto ao tipo de material e época de coleta. Tem sido usada, com maior freqüência, em vez da folha inteira, a análise do pecíolo ou limbo foliar (DAL BÓ, et. al. 1989). BERTONI; MORARD (1982), em estudo com a cultivar Chassellas, determinaram que o conteúdo de $\mathrm{N}$ dos limbos foliares foi duas vezes maior que o dos pecíolos, enquanto que para $\mathrm{P}, \mathrm{K}, \mathrm{Mg}$ e $\mathrm{Ca}$, os valores para limbo e pecíolo foram similares. A coleta pode ser feita em duas ocasiões: na floração, onde ocorre um pico de concentração da maioria dos nutrientes nos tecidos ou no início da troca de cor das bagas, quando há maior amplitude da época de coleta.

Para Tecchio et al. (2006), embora a prática da adubação seja realizada pela maioria dos viticultores no Brasil, eles a fazem de forma errônea, baseada em orientações empíricas, sem o conhecimento das reais condições do solo e das necessidades das plantas, utilizando os fertilizantes inadequadamente, ocasionando dessa maneira desequilíbrios nutricionais, o que acarreta queda na produção e na qualidade dos frutos. Dentre as inúmeras práticas culturais objetivando aumento em produtividade e na qualidade da uva, merece destaque a aplicação balanceada de nutrientes mediante adubações equilibradas, baseadas em análises químicas de solo e de folha.

Uma das grandes utilidades da diagnose foliar consiste no levantamento nutricional das lavouras, trazendo relevante contribuição quando os resultados da análise foliar são acompanhados dos de produção (MALAVOLTA; VITTI; OLIVEIRA, 1997). O levantamento dos teores foliares em vinhedos de baixa produtividade, quando comparado com os de alta produtividade, tem a finalidade de identificar a existência de deficiências e excessos de nutrientes para direcionar a programação de pesquisas regionais de adubação (TECCHIO et al., 2006).

O objetivo deste trabalho foi o de caracterizar o teor de nutrientes minerais da coleção de germoplasma da Universidade Estadual do Norte Fluminense, para subsidiar a implantação de um método de diagnose nutricional com base do limbo foliar, com vistas à recomendação de adubação vinhedos da região Norte e Noroeste Fluminense.

O experimento foi conduzido em uma coleção de germoplasma de videira instalado na área experimental do colégio Estadual Agrícola Antônio Sarlo, no município de Campos dos Goytacazes, na região Norte do Estado do Rio de Janeiro, situado a $21^{\circ} 45^{\prime} \mathrm{S}$ e $41^{\circ} 20^{\prime} \mathrm{W}$ e $11 \mathrm{~m}$ de altitude, classificado como tropical chuvoso, clima de bosque. A precipitação média anual é de $1.023 \mathrm{~mm}$, evapotranspiração potencial de $1.601 \mathrm{~mm}$ anuais e temperatura média anual de $23^{\circ} \mathrm{C}$. A área cultivada compreende $2.500 \mathrm{~m}^{2}$ com doze genótipos de uvas enxertadas sob o porta-enxerto IAC 572 'Jales', com 6 anos de idade, num solo de textura média a arenosa.

Odelineamento usado foi ode blocos casualizados com três repetições, sendo a unidade experimental composta por quatro plantas, espaçadas em $4 \mathrm{~m}$ x 3 $\mathrm{m}$, com sistema de condução em latada.

Foram avaliados doze genótipos de videira: 'Itália', 'Rubi', 'Patrícia', 'Isabel', 'Redglobe', 'Roberta', 'Kyoho', 'Romana', 'Rosalinda', 'Moscatel de Hamburgo', 'Niágara Rosada' e 'UVF 01'. Todos os genótipos foram obtidos do IAC, exceto 'UFV 01', cuja origem foi da Universidade Federal de Viçosa.

As amostragens para análises foliares foram realizadas em duas épocas do ciclo reprodutivo da 
videira: no pleno florescimento e início da maturação das bagas ("veraison").

Coletou-se, para análise, o limbo foliar de folhas recém-maduras, contadas a partir do ápice para a base, sendo opostas ao cacho (TERRA, 2003; TERRA et al., 2007). Retiraram-se cinco folhas por parcela experimental de cada genótipo para análise foliar.

Foram analisados os teores de nitrogênio $(\mathrm{N})$, fósforo $(\mathrm{P})$, potássio $(\mathrm{K})$, cálcio $(\mathrm{Ca})$, magnésio $(\mathrm{Mg})$, enxofre $(\mathrm{S})$, ferro $(\mathrm{Fe})$, zinco $(\mathrm{Zn})$, cobre $(\mathrm{Cu})$, cloro $(\mathrm{Cl})$, manganês $(\mathrm{Mn})$, boro $(\mathrm{B})$ e molibdênio (Mo). As análises foliares foram feitas de acordo com metodologia descrita por Malavolta, Vitti e Oliveira (1997) e Jones Júnior et al. (1991).

Os dados foram submetidos à análise de variância. Utilizou-se um esquema fatorial $2 \times 12$ (2 épocas de amostragem foliar x 12 genótipos), exceto o genótipo UFV01, que não floresceu no período de amostragem em razão das condições edafoclimáticas. Os graus de liberdade dos fatores isolados, assim como a interação, foram desdobrados via teste de comparação de médias, sendo utilizado o teste Tukey a $5 \%$ de probabilidade.

Observou-se que o teor foliar de $\mathrm{N}$ diferiu significativamente entre os genótipos estudados. $\mathrm{O}$ teor de $\mathrm{N}$ manteve-se dentro da faixa normal nas duas épocas de amostragem do ciclo: pleno florescimento e o início da maturação das bagas ("veraison”).

Um dos fatores que determinam nível nutricional de $\mathrm{N}$ encontrado nos genótipos desta coleção de germoplasma foram as adubações convencional e orgânica e os elevados níveis de precipitações pluviométricas, que normalmente ocorrem na região, que em outubro, novembro e dezembro de 2006 foram 121,8mm, 207,4mm e 84,4mm, respectivamente, e que devem ter contribuído para a boa absorção de $\mathrm{N}$ pelos genótipos de videira.

Com relação ao $\mathrm{K}$, a média do teor deste elemento manteve-se na faixa normal nas duas épocas amostradas do ciclo. O nível nutricional encontrado para $\mathrm{K}$ pode ser explicado pelos teores encontrado no solo da região Norte Fluminense e a adubação realizada no experimento. Cabe verificar que, conforme Terra et al. (2007), a época de pleno florescimento utilizada para coleta do limbo foliar adotada neste trabalho é a mais adequada para diagnóstico nutricional de $\mathrm{K}$.

Para o $\mathrm{P}$, nenhum genótipo apresentou nível abaixo da faixa normal (Tabela 1). Tal resultado parece ser explicado pela alta capacidade da videira, em extrair e utilizar eficientemente esse nutriente. Entretanto, esses dados não corroboram com os obtidos por Terra et al. (2007) na avaliação do estado nutricional da videira 'Itália' na região de São Miguel Arcanjo, SP.

Os genótipos apresentaram níveis de $\mathrm{Ca}$ abaixo do normal nas duas épocas de amostragem do ciclo. Esses resultados não estão de acordo com Terra et al. (2007). Para o Mg, a situação verificada foram teores superiores na primeira época de amostragem e inferior na fase de mudança da cor da baga. Nesta última época de amostragem, tais teores podem estar ocorrendo também devido aos altos níveis de $\mathrm{K}$, resultando numa menor absorção de $\mathrm{Ca}$ e $\mathrm{Mg}$.

O S apresentou média de 2,16 g. $\mathrm{Kg}^{-1}$ a 2,15 g. $\mathrm{Kg}^{-1}$. Esses teores são confirmados com os resultados obtidos por Fráguas e Czermwnski (2001) e Terra et al. (2007). 
Tabela 1. Teores médios dos macronutrientes N, P e K do limbo foliar dos genótipos de uva cultivados em Campos dos Goytacazes-RJ em pleno florescimento e na troca de cor da baga.

\begin{tabular}{|c|c|c|c|c|c|c|}
\hline \multirow[b]{3}{*}{ Genótipo } & \multicolumn{6}{|c|}{ Teores dos elementos no Limbo foliar (g. Kg $\left.{ }^{-1}\right)$} \\
\hline & \multicolumn{2}{|c|}{$\mathbf{N}$} & \multicolumn{2}{|c|}{$\mathbf{P}$} & \multicolumn{2}{|c|}{$\mathbf{K}$} \\
\hline & $\begin{array}{c}\text { Pleno } \\
\text { florescimento }\end{array}$ & $\begin{array}{c}\text { Troca de cor da } \\
\text { baga }\end{array}$ & $\begin{array}{c}\text { Pleno } \\
\text { florescimento }\end{array}$ & $\begin{array}{c}\text { Troca de cor } \\
\text { da baga }\end{array}$ & $\begin{array}{c}\text { Pleno } \\
\text { florescimento }\end{array}$ & $\begin{array}{c}\text { Troca de cor } \\
\text { da baga }\end{array}$ \\
\hline Redglobe & 21,50 a A & 24,10 a $\mathrm{A}$ & $2,20 \mathrm{bA}$ & 3,70 a $\mathrm{A}$ & 15,30 a $\mathrm{BC}$ & 13,30 a $\mathrm{A}$ \\
\hline Roberta & 22,60 a A & 21,60 a $\mathrm{A}$ & 2,60 a $\mathrm{A}$ & 2,00 a $\mathrm{BC}$ & $15,60 \mathrm{a} \quad \mathrm{B}$ & $12,00 \mathrm{~b} \mathrm{AB}$ \\
\hline Itália & 22,60 a A & 19,50 a $\mathrm{A}$ & 2,70 a A & 2,50 a $\mathrm{ABC}$ & 11,90 a $\mathrm{BCDEF}$ & 10,60 a $\mathrm{AB}$ \\
\hline N. Rosada & 24,10 a A & 19,10 b A & 1,90 a A & 2,10 a $\mathrm{ABC}$ & 14,70 a $\mathrm{BC}$ & 9,30 b AB \\
\hline Rosalinda & 20,40 a A & 21,40 a $\mathrm{A}$ & 1,90 a A & 1,80 a $\mathrm{C}$ & $8,50 \mathrm{a}$ & $7,50 \mathrm{a} \quad \mathrm{B}$ \\
\hline Isabel & 20,70 a A & 21,60 a A & 1,80 a $\mathrm{A}$ & 1,80 a $\mathrm{ABC}$ & 9,10 a $\quad$ DEF & $9,10 \mathrm{a} \quad \mathrm{AB}$ \\
\hline Patrícia & 26,30 a A & $21,80 \mathrm{~b} \mathrm{~A}$ & $2,20 \mathrm{a} \mathrm{A}$ & 1,90 a $\mathrm{BC}$ & $7,10 \mathrm{a}$ & 9,60 a $\mathrm{AB}$ \\
\hline Rubi & 21,60 a $\mathrm{A}$ & 21,80 a $\mathrm{A}$ & 1,70 a $\mathrm{A}$ & 1,90 a $\mathrm{BC}$ & 10,50 a $\mathrm{CDEF}$ & $12,30 \mathrm{a} \quad \mathrm{AB}$ \\
\hline Romana & 24,60 a $\mathrm{A}$ & $20,40 \mathrm{~b} \mathrm{~A}$ & 2,10 a $\mathrm{A}$ & 2,00 a $\mathrm{ABC}$ & 10,50 a $\mathrm{CDEF}$ & 13,00 a $\mathrm{A}$ \\
\hline Kyoho & 21,70 a $\mathrm{A}$ & 21,10 a $\mathrm{A}$ & $2,30 \mathrm{~b} \mathrm{~A}$ & 3,50 a $\mathrm{AB}$ & 13,20 a $\mathrm{BCDE}$ & 13,50 a $A$ \\
\hline M. Hamburgo & 22,70 a A & 22,90 a $\mathrm{A}$ & $2,20 \mathrm{~b} \mathrm{~A}$ & 3,20 a $\mathrm{ABC}$ & 13,60 a $\mathrm{BCD}$ & $13,40 \mathrm{a} \quad \mathrm{A}$ \\
\hline UFV 01 & 24,80 a A & - & $2,40 \mathrm{~b} \mathrm{~A}$ & - & 20,80 a A & - \\
\hline Média & 22,81 & 21,00 & 2,16 & 13,27 & 12,57 & 3,45 \\
\hline CV (\%) & $7,85 \%$ & $8,30 \%$ & $14,61 \%$ & $28,50 \%$ & $30,14 \%$ & $18,64 \%$ \\
\hline
\end{tabular}

Médias seguidas pela mesma letra, minúscula na linha e maiúscula na coluna, não diferem entre si, a 5\% de probabilidade, pelo teste de Tukey.

Tabela 2. Teores médios dos macronutrientes $\mathrm{S}$, Ca e $\mathrm{Mg}$ do limbo foliar $\left(\mathrm{g} \mathrm{Kg}^{-1}\right)$ dos genótipos de uva cultivados em Campos dos Goytacazes-RJ em pleno florescimento e na troca de cor da baga.

\begin{tabular}{|c|c|c|c|c|c|c|}
\hline \multirow[b]{3}{*}{ Genótipo } & \multicolumn{6}{|c|}{ Teores dos elementos no Limbo foliar (g. $\mathrm{Kg}^{-1}$ ) } \\
\hline & \multicolumn{2}{|c|}{$\mathbf{S}$} & \multicolumn{2}{|c|}{$\mathbf{C a}$} & \multicolumn{2}{|c|}{ Mg } \\
\hline & $\begin{array}{c}\text { Pleno } \\
\text { florescimento }\end{array}$ & $\begin{array}{c}\text { Troca de cor } \\
\text { da baga }\end{array}$ & $\begin{array}{c}\text { Pleno } \\
\text { florescimento }\end{array}$ & $\begin{array}{c}\text { Troca de } \\
\text { cor da baga }\end{array}$ & $\begin{array}{c}\text { Pleno } \\
\text { florescimento }\end{array}$ & $\begin{array}{c}\text { Troca de cor da } \\
\text { baga }\end{array}$ \\
\hline Redglobe & $1,80 \mathrm{a} \mathrm{A}$ & 1,90 a $\mathrm{A}$ & 9,00 a $\mathrm{BC}$ & 13,80 a $\mathrm{AB}$ & 3,20 a $\mathrm{BCDE}$ & 3,80 a $\mathrm{ABC}$ \\
\hline Roberta & 1,90 a $\mathrm{A}$ & 1,80 a A & 8,00 a $\mathrm{BC}$ & 10,30 a $\mathrm{AB}$ & 3,10 a $\quad \mathrm{CDE}$ & 3,30 a $\mathrm{BCD}$ \\
\hline Itália & 2,10 a $\mathrm{A}$ & 2,80 a $\mathrm{A}$ & $7,00 \mathrm{a} \quad \mathrm{C}$ & 7,30 a $\mathrm{B}$ & $2,40 \mathrm{~b} \quad \mathrm{DE}$ & 3,10 a $\mathrm{AB}$ \\
\hline N. Rosada & 2,10 a $\mathrm{A}$ & 2,20 a A & $12,00 \mathrm{bABC}$ & 16,20 a A & $3,50 \mathrm{~b}$ BCD & 4,30 a $\mathrm{AB}$ \\
\hline Rosalinda & 2,50 a $\mathrm{A}$ & $2,20 \mathrm{a} \mathrm{A}$ & $14,00 \mathrm{a} A B$ & 12,70 a $\mathrm{AB}$ & 4,40 a $\mathrm{AB}$ & 3,90 a $\mathrm{BCD}$ \\
\hline Isabel & 2,30 a $\mathrm{A}$ & 2,50 a A & 12,40 a $\mathrm{ABC}$ & 13,70 a $\mathrm{AB}$ & 3,30 a BDDE & 3,40 a A \\
\hline Patrícia & 2,90 a $\mathrm{A}$ & 2,60 a $A$ & 18,90 a A & 15,70 a A & 5,00 a $\mathrm{A}$ & 4,80 a $\mathrm{A}$ \\
\hline Rubi & 2,30 a $\mathrm{A}$ & 2,20 a A & 12,90 a $\mathrm{ABC}$ & 14,80 a $\mathrm{A}$ & 3,90 a $\mathrm{ABC}$ & 4,00 a $\mathrm{AB}$ \\
\hline Romana & 2,50 a $\mathrm{A}$ & 2,10 a $\mathrm{A}$ & 12,90 a $\mathrm{ABC}$ & 14,80 a $\mathrm{AB}$ & 3,00 a $\quad \mathrm{CDE}$ & 2,60 a $\quad C D$ \\
\hline Kyoho & 1,80 a $\mathrm{A}$ & 1,70 a $\mathrm{A}$ & 12,50 a $\mathrm{BC}$ & 11,00 a $\mathrm{AB}$ & $2,50 \mathrm{a} \quad \mathrm{DE}$ & $2,50 \mathrm{a} \quad \mathrm{D}$ \\
\hline M. Hamburgo & 1,90 a $\mathrm{A}$ & 1,80 a $\mathrm{A}$ & 8,00 a $\mathrm{BC}$ & 10,20 a $\mathrm{AB}$ & 3,30 a $\mathrm{BCDE}$ & 3,50 a $\mathrm{BCD}$ \\
\hline UFV 01 & 1,90 a $\mathrm{A}$ & - & 7,10 a $\mathrm{C}$ & - & $2,30 \mathrm{a}$ & - \\
\hline Média & 2,16 & 2,15 & 11,08 & 12,58 & 3,32 & 3,56 \\
\hline CV (\%) & $15,74 \%$ & $16,35 \%$ & $31,43 \%$ & $21,21 \%$ & $24,23 \%$ & $19,30 \%$ \\
\hline
\end{tabular}

Médias seguidas pela mesma letra, minúscula na linha e maiúscula na coluna, não diferem entre si, a 5\% de probabilidade, pelo teste de Tukey. 
Verificam-se, na (Tabela 3), os valores elevados de $\mathrm{Cu}$, Mn e Zn são justificáveis pela contaminação das folhas pelos fungicidas empregados, mas sem causar fitotoxidez, por estarem mais na superfície foliar e não em nível celular. Esses resultados corroboram com os obtidos por Fráguas e Czermainski (2001) e Terra (2007).
Para o $\mathrm{B}$, os genótipos apresentaram faixas normais (Tabela 3) com concentração variando de 44,97 mg. $\mathrm{Kg}^{-1}$ a $54,14 \mathrm{mg}$. $\mathrm{Kg}^{-1}$. Fráguas e Czermainski (2001), Tecchio et al. (2007) e Terra et al. (2007) encontraram resultados inferiores que os apresentados neste trabalho.

Tabela 3. Teores médios dos macronutrientes $\mathrm{Mn}, \mathrm{Zn}$ e $\mathrm{Cu}$ do limbo foliar dos genótipos de uva cultivados em Campos dos Goytacazes-RJ em pleno florescimento e na troca de cor da baga.

\begin{tabular}{|c|c|c|c|c|c|c|}
\hline \multirow[b]{3}{*}{ Genótipo } & \multicolumn{6}{|c|}{ Teores dos elementos no Limbo foliar (g. $\left.\mathrm{Kg}^{-1}\right)$} \\
\hline & \multicolumn{2}{|c|}{ Mn } & \multicolumn{2}{|c|}{$\mathbf{Z n}$} & \multicolumn{2}{|c|}{$\mathbf{C u}$} \\
\hline & $\begin{array}{c}\text { Pleno } \\
\text { florescimento } \\
\end{array}$ & $\begin{array}{c}\text { Troca de cor } \\
\text { da baga }\end{array}$ & $\begin{array}{c}\text { Pleno } \\
\text { florescimento }\end{array}$ & $\begin{array}{c}\text { Troca de cor } \\
\text { da baga }\end{array}$ & $\begin{array}{c}\text { Pleno } \\
\text { florescimento } \\
\end{array}$ & $\begin{array}{c}\text { Troca de cor da } \\
\text { baga }\end{array}$ \\
\hline Redglobe & $247,90 \mathrm{aA}$ & $250,80 \mathrm{aAB}$ & $46,80 \mathrm{a} B C$ & $46,50 \mathrm{a} \quad \mathrm{D}$ & $541,90 \mathrm{a} \mathrm{A}$ & $287,20 \mathrm{a} \mathrm{A}$ \\
\hline Roberta & $244,00 \mathrm{aA}$ & $179,40 \mathrm{aAB}$ & $46,70 \mathrm{a} \mathrm{BC}$ & $49,60 \mathrm{a} \quad \mathrm{D}$ & $624,10 \mathrm{a} \mathrm{A}$ & $620,30 \mathrm{a} \mathrm{A}$ \\
\hline Itália & $284,90 \mathrm{aA}$ & $215,50 \mathrm{aAB}$ & $55,70 \mathrm{a} \mathrm{ABC}$ & $54,20 \mathrm{a} \quad \mathrm{CD}$ & $625,70 \mathrm{aA}$ & $396,30 \mathrm{a} \mathrm{A}$ \\
\hline N. Rosada & $367,80 \mathrm{aA}$ & 428,20 a $\mathrm{A}$ & $58,50 \mathrm{a} \mathrm{ABC}$ & $59,50 \mathrm{a} \quad \mathrm{CD}$ & $529,10 \mathrm{a} \mathrm{A}$ & $369,00 \mathrm{a} \mathrm{A}$ \\
\hline Rosalinda & $394,80 \mathrm{aA}$ & 347,10 a $\mathrm{A}$ & $69,70 \mathrm{a} A B$ & $79,30 \mathrm{a} \quad \mathrm{BCD}$ & $435,90 \mathrm{a} \mathrm{A}$ & $487,50 \mathrm{a} A$ \\
\hline Isabel & $408,10 \mathrm{aA}$ & 452,00 a A & $74,30 \mathrm{a} A B$ & 91,00 a $B C$ & $692,80 \mathrm{a} \mathrm{A}$ & $462,60 \mathrm{a} A$ \\
\hline Patrícia & $526,10 \mathrm{aA}$ & 347,00 a A & $92,30 \mathrm{a} \mathrm{A}$ & $59,50 \mathrm{a} \quad \mathrm{CD}$ & 761,90a A & $462,60 \mathrm{a} A$ \\
\hline Rubi & $392,90 \mathrm{aA}$ & 363,50 a $\mathrm{A}$ & $63,70 \mathrm{a} \mathrm{ABC}$ & $134,70 \mathrm{a} \mathrm{A}$ & $518,30 \mathrm{a} A$ & $431,30 \mathrm{a} A$ \\
\hline Romana & $352,10 \mathrm{aA}$ & $307,10 \mathrm{aAB}$ & $59,60 \mathrm{a} \mathrm{ABC}$ & 99,10 a $\mathrm{A}$ B & $502,80 \mathrm{a} \mathrm{A}$ & $494,50 \mathrm{a} A$ \\
\hline Kyoho & $202,10 \mathrm{aA}$ & $195,20 \mathrm{aAB}$ & $50,60 \mathrm{~b}$ BC & $135,00 \mathrm{a} A$ & $624,90 \mathrm{a} \mathrm{A}$ & $567,50 \mathrm{a} A$ \\
\hline M. Hamburgo & $308,50 \mathrm{aA}$ & $237,40 \mathrm{aAB}$ & $53,80 \mathrm{a}$ BC & $89,90 \mathrm{a} \quad$ BC & $675,50 \mathrm{a} A$ & $411,40 \mathrm{a} \mathrm{A}$ \\
\hline UFV 01 & $400,50 \mathrm{~A}$ & - & $29,80 \quad \mathrm{C}$ & - & $421,80 \mathrm{~A}$ & - \\
\hline Média & 344,13 & 302,13 & 58,36 & 81,57 & 579,63 & 401,51 \\
\hline CV (\%) & $26,31 \%$ & $30,87 \%$ & $27,11 \%$ & $38,97 \%$ & $18,06 \%$ & $37,58 \%$ \\
\hline
\end{tabular}

Médias seguidas pela mesma letra, minúscula na linha e maiúscula na coluna, não diferem entre si, a 5\% de probabilidade, pelo teste de Tukey.

$\mathrm{O}$ Fe apresentou teores nas faixas normais nas três épocas amostradas do ciclo. Porém, os teores apresentados neste trabalho são inferiores aos encontrados por Fráguas e Czermainski (2001), Tecchio et al. (2007) e Terra et al. (2007).
O Mo apresentou média de 0,21 mg. $\mathrm{Kg}^{-1}$ a 0,33 mg. $\mathrm{Kg}^{-1}$, sendo que houve um decréscimo dos teores foliares da primeira época de amostragem as outras duas épocas de amostragem. 
Tabela 4. Teores médios dos macronutrientes $\mathrm{Mn}, \mathrm{Zn}$ e $\mathrm{Cu}$ do limbo foliar dos genótipos de uva cultivados em Campos dos Goytacazes-RJ em pleno florescimento e na troca de cor da baga.

\begin{tabular}{|c|c|c|c|c|c|c|}
\hline \multirow[b]{3}{*}{ Genótipos } & \multicolumn{6}{|c|}{ Teores dos elementos no Limbo foliar (mg. $\left.\mathrm{Kg}^{-1}\right)$} \\
\hline & \multicolumn{2}{|c|}{ Mo } & \multicolumn{2}{|c|}{$\mathrm{Fe}$} & \multicolumn{2}{|c|}{ B } \\
\hline & $\begin{array}{c}\text { Pleno } \\
\text { florescimento }\end{array}$ & $\begin{array}{c}\text { Troca de cor } \\
\text { da baga }\end{array}$ & $\begin{array}{c}\text { Pleno } \\
\text { florescimento }\end{array}$ & $\begin{array}{l}\text { Troca de cor } \\
\text { da baga }\end{array}$ & $\begin{array}{c}\text { Pleno } \\
\text { florescimento }\end{array}$ & $\begin{array}{l}\text { Troca de cor } \\
\text { da baga }\end{array}$ \\
\hline Redglobe & $0,40 \mathrm{a} A$ & $0,40 \mathrm{aA}$ & 94,10 a $\mathrm{BC}$ & $90,60 \mathrm{a} A B$ & 53,00 a $\mathrm{B}$ & 59,00 a A \\
\hline Roberta & $0,20 \mathrm{aA}$ & $0,10 \mathrm{aA}$ & 74,20 a $\mathrm{C}$ & $79,50 \mathrm{a} A B$ & 43,10 a $\mathrm{B}$ & 49,60 a A \\
\hline Itália & $0,10 \mathrm{a} A$ & $0,50 \mathrm{aA}$ & 69,10 a $\mathrm{BC}$ & $109,30 \mathrm{a} A$ & 43,60 a $\mathrm{B}$ & 46,70 a A \\
\hline N. Rosada & $0,20 \mathrm{a} A$ & $0,20 \mathrm{aA}$ & 94,30 a $\mathrm{BC}$ & $133,10 \mathrm{a} \mathrm{A}$ & 58,20 a $\mathrm{B}$ & $38,80 \mathrm{a} \mathrm{A}$ \\
\hline Rosalinda & $0,20 \mathrm{a} A$ & $0,30 \mathrm{aA}$ & $133,00 \mathrm{a} A B C$ & $116,50 \mathrm{a} A$ & 45,90 a $\mathrm{B}$ & 39,80 a A \\
\hline Isabel & $0,30 \mathrm{a} A$ & $0,20 \mathrm{aA}$ & $107,20 \mathrm{a} \mathrm{BC}$ & $122,80 \mathrm{a} A$ & 45,70 a $\mathrm{B}$ & 40,50 a $\mathrm{A}$ \\
\hline Patrícia & $0,20 \mathrm{a} A$ & $0,20 \mathrm{aA}$ & $183,00 \mathrm{a} A B$ & $153,70 \mathrm{a} A$ & $154,50 \mathrm{a} A$ & $62,30 \mathrm{~b} \mathrm{~A}$ \\
\hline Rubi & $0,20 \mathrm{a} A$ & $1,10 \mathrm{aA}$ & $112,20 \mathrm{a}$ BC & $126,90 \mathrm{a} A$ & 35,30 a $\mathrm{B}$ & 45,90 a $\mathrm{A}$ \\
\hline Romana & $0,30 \mathrm{a} A$ & $0,30 \mathrm{aA}$ & $107,70 \mathrm{a}$ BC & 91,60 a $\mathrm{A}$ & 52,00 a $\mathrm{B}$ & 31,60 a $A$ \\
\hline Kyoho & $0,20 \mathrm{a} A$ & $0,20 \mathrm{aA}$ & 81,90 a $\mathrm{C}$ & 94,80 a A & 39,30 a $\mathrm{B}$ & 37,20 a $\mathrm{A}$ \\
\hline M. Hamburgo & $0,20 \mathrm{a} A$ & $0,30 \mathrm{aA}$ & 84,70 a $\mathrm{C}$ & $130,60 \mathrm{a} A$ & 47,20 a $\mathrm{B}$ & 43,50 a $\mathrm{A}$ \\
\hline UFV 01 & $0,20 \mathrm{~A}$ & - & $205,60 \mathrm{~A}$ & - & 31,90 a $B$ & - \\
\hline Média & 0,21 & 0,33 & 112,23 & 113,57 & 54,14 & 44,97 \\
\hline CV (\%) & $35,28 \%$ & $79,06 \%$ & $27,11 \%$ & $38,97 \%$ & $18,06 \%$ & $37,58 \%$ \\
\hline
\end{tabular}

Médias seguidas pela mesma letra, minúscula na linha e maiúscula na coluna, não diferem entre si, a 5\% de probabilidade, pelo teste de Tukey.

Cabe ressaltar que os métodos Conradie e Terblanche (1980) e Nogueira et al. (1992) recomendam proceder a adubação de manutenção no caso de o índice de balanço situa-se na faixa insuficiente ou abaixo da faixa normal. Ainda, recomendam a adoção de medidas quando os índices indicarem excesso, sendo assim, para as situações abaixo ou acima da faixa normal, os autores recomendam acompanhar a evolução do estado nutricional dos genótipos.

O aprimoramento do diagnóstico nutricional dos genótipos estudados, bem como de outros genótipos de importância econômica, trará para região a possibilidade de realizar adubação mais racional.

Conclui-se que: a) o limbo foliar coletado refletiu bem o estado nutricional dos genótipos de videira; b) os genótipos não apresentaram níveis de $\mathrm{N}$ abaixo do normal, sendo a maioria dos genótipos apresentaram níveis normais; c) nenhum genótipo apresentou níveis abaixo do normal de $\mathrm{K}$ e P; d) todos os genótipos apresentaram níveis de Ca abaixo da faixa normal para todas as épocas amostradas e o $\mathrm{Mg}$ na faixa normal; e) todos os genótipos apresentaram níveis normais de $\mathrm{B}$.

\section{Referências}

BERTONI, G; MORARD, P. Blade or petiole analysis as a guide for grape nutrition. Communications in soil science and plant analysis, New York, v. 13, n. 8, p. 598605, 1982.

CONRADIE, W. J. Norms for leaf analysis of vines. Stellenbosch: Department of Agriculture and Water Supply, 1986, 2 p.

CONRADIE, W. J.; TERBLANCHE, J. H. Leaf analysis of deciduos fruit trees and grapevines-summer rainfall area. Pretoria: Departamento Agricultural Technical Services, 1980. 2 p. (Table grapes: Summer Rainfall, G. 4).

DAL BÓ, M. A.; BECKER, M.; BASSO, C.; STUKER, H. Levantamento do Estado Nutricional da videira em Santa Catarina por análise de solo e tecido. R. Bras. Ci. Solo, Campinas, v. 13, p. 335-340, 1989. 
FRÁGUAS, J. C.; CZERMAINSKI, A. B. C. Avaliação de produtos para a nutrição da videira via foliar. R. Bras. Ci. Solo, Viçosa, MG, v. 25, n. 25, p.1007-10015, 2001.

JONES JúNIOR, J. B.; WOLF, B.; MIELS, H. A plants analysis handbook: a practical sampling, preparation, analysis, and interpretation guide. Athens (USA): MicroMacro Publishing, 1991, p. 213.

MALAVOLTA, E.; VITTI, G. C.; OLIVEIRA, S. A. de. Avaliações do estado nutricional das plantas: princípios e aplicações. 2. ed. Piracicaba: POTAFOS, 1997, p. 319. NOGUEIRA, D. J. P.; ABRAÃO, E.; CHALFUN, N. N. J.; ALVARENGA, A. A.; FRÁGUAS, J. C. Diagnóstico foliar com recursos aos balanços percentuais. Ci. Prát., Lavras, v. 16, n. 16, p. 25-30, 1992.

TECCHIO, M. A.; PAIOLI-PIRES, E. J.; TERRA, M. M.; GRASSI FILHO, H.; CORREAA, J. C.; VIEIRA, C. R. Y. Correlação entre a produtividade e os resultados de análise foliar e de solo em vinhedos de Niagara Rosada. Ciênc. Agrotec., Lavras, v. 30, n. 6, p. 1056-1064, 2006.

TECCHIO, M. A.; PAIOLI-PIRES, E. J.; TERRA, M. M.; MOURA, M. F. Produtividade e teores de nutrientes da videira 'Niagara Rosada' em vinhedos nos municípios de Louveira e Jundiaí. Biosci. J., Uberlândia, v. 23, n. 1, p. 48-58, 2007.

TERRA, M. M. Nutrição, calagem e adubação. In: POMMER, C. V. (Ed.). Uva: tecnologia de produção, pós-colheita, mercado. Porto Alegre: Cinco Continentes, 2003. p. 405-476.

TERRA, M. M.; GERGOLETTI, I. F.; PIRES, E. J. P.; BOTELHO, R. V.; SANTOS, W. R. dos; TECCHIO, M. A. Avaliação do estado nutricional da videira 'Itália' na região de São Miguel Arcanjo-SP, utilizando o sistema integrado de diagnose e recomendação. Rev. Bras. Frutic., Cruz das Almas, v. 29, n. 3, p. 710-716, 2007. 
